\title{
ANNOUNCEMENT
}

\section{Call for Papers: Special Issue on Big Data in Robotics}

Guest Editors: Jeannette Bohg, PhD, Matei Ciocarlie, PhD, Javier Civera, PhD, and Lydia E. Kavraki, PhD

\section{Deadline for submission: June 15, 2016}

Big Data and Robotics appear to be on a perfect convergence path. For a number of years, one of the main challenges in Robotics has been moving out of perfectly structured environments and into the "real world," characterized by its incredible range of possible tasks and situations. From autonomous cars to mobile manipulators, new big data methods have the potential to allow robots to understand and operate in significantly more complex environments than was possible even in the recent past. At the same time, robots are embodied agents that have the possibility to interact with the environment. Thereby, they can collect far richer data sets than traditional, strictly passive or even active (viewpoint-changing) sensors. Such a virtuous cycle could lead to quantum leaps in robotics and big data. How far along are we on the way of fulfilling this seemingly great potential? What has been achieved, and what great challenges lie ahead?

This Big Data journal special issue on Robotics, scheduled for publication in December of 2016, will showcase research in which robotics and big data converge to solve new problems and demonstrate applicability to new domains. The special issue has a primary aim to describe and discuss:

1. Successful examples of using big data in robotics to achieve new capabilities and operate in increasingly more complex environments.

2. The use of robots as sensor platforms that interact with the world and collect rich, multimodal data sets, containing features and patterns relevant to robotic tasks and beyond.

3. The interplay between sensing and action, at a large scale and with real-world applications.

4. The tradeoff between purely data-driven methods, potentially requiring very large training datasets, and model-driven approaches, relying on prior knowledge and existing problem structure.

The deadline for manuscript submission is June 15, 2016. Please submit your papers online to our web-based manuscript submission and peer-review at: www.liebertpub.com/manuscript/big

Visit our website to read about the Journal and to view a sample issue: www.liebertpub. $\mathrm{com} / \mathrm{big}$

Big Data is a highly innovative, peer-reviewed journal that provides a unique forum for worldclass research exploring the challenges and opportunities in collecting, analyzing, and disseminating vast amounts of data, including data science, big data infrastructure and analytics, and pervasive computing. There are many advantages to publishing in Big Data, including: 
- Indexed in Thomson Reuters Web of Science ${ }^{\mathrm{TM}}$ Science Citation Index Expanded (SciSearch) and Journal Citation Reports/Science Edition

- Fast and user-friendly electronic submission

- Rapid, high-quality peer review

- Maximum exposure: accessible in 170 countries worldwide

\section{Editorial questions?}

Contact Guest Editor Dr. Lydia E. Kavraki at kavraki@rice.edu

\section{Manuscript preparation or technical questions?}

Contact authorservices@liebertpub.com

Cite this article as: Bohg J, Ciocarlie M, Civera J, Kavraki LE (2016)

Call for papers: Special issue on big data in robotics. Big Data 4:1, 1-2,

DOI: 10.1089/big.2016.29005.cfp. 